\title{
Civilisations
}

Revue internationale d'anthropologie et de sciences

humaines

42-1 | 1993

Amérique latine-Europe

\section{Les relations économiques entre l'Amérique latine et la communauté européenne : internationalisation, régionalisation et coopération}

\section{Marc Rimez}

\section{(2) OpenEdition}

\section{Journals}

Édition électronique

URL : http://journals.openedition.org/civilisations/2107

DOI : 10.4000/civilisations. 2107

ISSN : 2032-0442

Éditeur

Institut de sociologie de l'Université Libre de Bruxelles

\section{Édition imprimée}

Date de publication : 1 novembre 1993

Pagination : 161-190

ISBN : 0009-8140

ISSN : 0009-8140

Référence électronique

Marc Rimez, «Les relations économiques entre l'Amérique latine et la communauté européenne internationalisation, régionalisation et coopération », Civilisations [En ligne], 42-1 | 1993, mis en ligne le 30 novembre 1996, consulté le 02 mai 2019. URL : http://journals.openedition.org/civilisations/2107 ; DOI : 10.4000/civilisations.2107

Ce document a été généré automatiquement le 2 mai 2019.

(c) Tous droits réservés 


\title{
Les relations économiques entre l'Amérique latine et la communauté européenne : internationalisation, régionalisation et coopération
}

\author{
Marc Rimez
}

1 La convergence de deux événements à caractère historique et éminemment politique, la célébration du cinquième centenaire de la date fétiche de 1492 d'une part, et la création du Marché unique européen, d'autre part, a stimulé l'étude d'un phénomène souvent laissé dans l'ombre durant les dernières décennies: les différents aspects des relations entre l'Amérique latine et l'Europe. Dans le domaine économique, en particulier, cet intérêt rénové s'est traduit par la réalisation et la publication d'un certain nombre d'études et d'articles qui permettent d'avoir maintenant une vue relativement approfondie de ces relations.

2 L'objet du présent article consistera donc moins à présenter une analyse détaillée des relations économiques, commerciales et financières entre l'Amérique latine et la Communauté Européenne (CE) qu'à en proposer une interprétation dans le cadre des grandes tendances qui ont marqué l'évolution de l'économie mondiale durant la décennie des années 80 . Sur cette base, on tentera de dégager les grandes lignes qui conforment la dynamique des relations économiques entre les deux régions et d'établir les points de divergence et de convergence dans l'agenda futur de ces relations.

3 Auparavant, et au risque de répéter une fois de plus ce qu'on a écrit récemment, il est utile de rappeler brièvement le contexte historique qui a pour le moins influencé, sinon conditionné, l'évolution récente des relations économiques et politiques entre l'Amérique latine et les pays européens. Il ne s'agit point ici d'entrer dans la polémique sur les vices et les vertus supposés de la colonisation, ni dans le faux débat sur l'importance relative des facteurs externes et des facteurs internes dans l'évolution économique et sociale de l'Amérique latine et son processus de développement ou de sous-développement. 


\section{Survol historique}

4 Les formes concrètes, prises au cours de l'Histoire, des structures économiques et sociales de l'Amérique latine sont le résultat d'une dialectique permanente entre des modalités d'insertion dans l'économie internationale et la capacité de réaction et d'adaptation des structures internes aux chocs externes. Il est indéniable que l'Europe et les Européens sont à l'origine de la plupart de ces chocs externes, tout au moins jusqu'au début du vingtième siècle.

Dans cette perspective, la première phase de la colonisation de l'Amérique latine par l'Espagne, basée sur le pillage et l'exploitation systématique des mines de métaux précieux (jusque vers 1650), signifie une incorporation brutale d'importants territoires, ressources et populations, à une économie internationale qui est en phase de constitution. Dès le départ, l'Amérique latine est insérée dans l'économie internationale à une place et avec une fonction bien spécifique, comme source de transfert d'excédents économiques, sous la forme, durant cette période, de l'exportation de métaux précieux. En même temps, les structures internes économiques et sociales sont réorganisées par la métropole en fonction de ces transferts d'excédents.

Durant tout le $18^{\text {ème }}$ siècle, les structures internes propres à la colonisation, en particulier la propriété foncière, se renforcent, tandis que les règles de monopole et d'exclusivité qui régissaient le commerce extérieur de l'Amérique latine au profit de l'Espagne, et, dans une moindre mesure, du Portugal, sont progressivement mises en brèche sous la pression convergente des intérêts des nouvelles puissances commerciales européennes en particulier l'Angleterre, et des groupes créoles locaux. Ainsi, ce n'est que vers la fin de ce siècle que l'interdiction faite aux colonies de commercer entre elles sera levée par la couronne espagnole.

7 Lors des guerres d'indépendance qui embraseront toute la région, à l'exception du Brésil, on retrouvera cette même convergence d'intérêts entre l'Angleterre, puissance hégémonique mondiale, et une partie des élites locales latino-américaines. L'Angleterre participera directement ou indirectement aux guerres d'indépendance, en particulier à travers leur financement, soucieux d'assurer ses intérêts économiques, elle exigera systématiquement le libre accès aux ports latino-américains, en échange de la reconnaissance diplomatique des nouveaux pays. Elle jouera aussi un rôle non négligeable à travers des interventions, pas seulement diplomatiques, dans le processus de balcanisation de la région qui consacrera le triomphe des intérêts locaux face aux tentatives intégrationnistes de quelques grands visionnaires latino-américains de l'époque. L'intensité des liens économiques et politiques avec la nouvelle métropole sera un des facteurs qui influenceront la place des nouvelles nations dans la division internationale du travail du $19^{\text {ème }}$ siècle.

8 La consolidation de la révolution industrielle en Europe, la demande croissante de matières premières et produits élémentaires que celle-ci entraîna, la réduction brutale des coûts de transport, provoqueront une réinsertion de la plupart des économies latinoaméricaines dans le noyau dynamique de l'économie mondiale. Ces économies sont alors intégrées comme fournisseurs de matières premières pour l'industrie européenne et de produits alimentaires dont la demande croît avec l'élévation du revenu réel moyen européen. En même temps, ces économies servent de débouchés aux produits manufacturés des industries européennes. Durant toute cette période de croissance 
extravertie, basée sur l'exportation des produits primaires, les structures économiques et sociales des pays latino-américains enregistreront des changements souvent substantiels, induisant dans certains cas une diversification socioéconomique et dans d'autres cas, le simple développement de ce qu'on a mal appelé les « économies d'enclave ». Durant cette période qui se termine avec la grande crise de 1929, les relations entre l'Europe et l'Amérique latine correspondent si bien au schéma classique de la division internationale du travail traditionnelle que l'on peut croire que celles-ci ont en fait servi de base empirique pour l'élaboration du modèle théorique.

Ce bref survol montre cependant que les traits fondamentaux de ce schéma - exportation de matières premières, accumulation extravertie et transferts d'excédents de la périphérie vers le centre - ont caractérisé sous des formes légèrement différentes suivant les époques le modèle d'insertion de l'Amérique latine dans l'économie internationale, qui s'est longtemps limitée à ses relations économiques avec l'Europe. On ne devra donc guère s'étonner de retrouver certains de ces caractères, bien sûr sous de nouvelles formes, dans les relations économiques actuelles entre les deux régions.

10 A travers la colonisation espagnole et portugaise d'abord, l'hégémonie commerciale, industrielle et financière anglaise ensuite, l'Europe a largement contribué à façonner non seulement les formes d'insertion de l'Amérique latine dans l'économie mondiale, mais aussi les structures internes économiques, politiques et sociales de ces pays durant près de quatre siècles. En termes historiques, l'interventionnisme nord-américain dans cette région ne s'est exercé que pendant un peu plus d'un siècle et demi, fondamentalement en Amérique centrale, y compris le Mexique, et dans les Caraïbes, tandis que l'hégémonie nord-américaine ne s'étendra à l'ensemble du sous-continent qu'à partir de la première guerre mondiale.

11 La pénétration économique européenne en Amérique latine aura marqué d'autant plus profondément les structures économiques de celle-ci - y compris sa «mentalité économique »- qu'elle ne s'est pas limitée à une simple domination étrangère ni à des apports massifs de capitaux et de technologie. Il convient de noter le rôle particulier qu'ont joué les vagues successives d'immigration européenne jusqu'à des périodes très récentes. Cette immigration sera généralement bien intégrée, avec des exceptions notables telles le Pérou, et, à travers le métissage, contribuera à la naissance de nouveaux peuples qui sauront construire et affirmer leur identité propre. Leurs "racines" européennes constituent probablement un des facteurs explicatifs des relations « privilégiées » qui continuent à exister entre les pays des deux régions, particulièrement sur les plans culturels et politiques.

Durant tout l'après-guerre, tandis que s'affirmait l'hégémonie nord-américaine dans l'économie mondiale, les relations économiques entre l'Amérique latine et l'Europe n'ont cessé de décroître en termes relatifs, tout en maintenant une structure asymétrique plus proche des formes traditionnelles de la division internationale du travail que de celle correspondant aux nouvelles données de l'économie internationale.

\section{Les grandes tendances de l'économie mondiale dans les années 80}

13 Les changements enregistrés dans le mode de fonctionnement et de régulation de l'économie internationale, et du système de relations internationales en général, ont eu 
un impact particulièrement accentué sur l'économie latino-américaine durant la dernière décennie. Suite à ces changements dans la sphère internationale d'une part, et à l'épuisement $\mathrm{du}$ modèle de développement basé sur l'import-substitution, artificiellement prolongé durant les années 70 à travers l'endettement externe, d'autre part, ces pays affrontent pour l'instant une période de redéfinition d'un certain nombre de paramètres fondamentaux de leur stratégie de développement, tels que le modèle d'industrialisation, le rôle de l'État et en particulier les modalités d'insertion internationale.

14 1. Une première tendance de fond se réfère à l'accélération de l'internationalisation de l'économie. Celle-ci s'exprime entre autres par :

- une intensification du commerce international de biens et surtout, de plus en plus, de services ;

- l'internationalisation de la production, en particulier à travers les entreprises transnationales (celles-ci représentaient $16,5 \%$ de la production manufacturière en 1988, contre $13,2 \%$ en 1973 ; cette proportion devrait atteindre $24 \%$ en $2000^{1}$ );

- l'internationalisation de la technologie, entre autres à travers les standardisations actuellement en cours dans les secteurs les plus dynamiques, tels que les télécommunications et l'informatique et la diffusion de la robotisation dans un nombre croissant de secteurs productifs ;

- la consolidation d'un modèle universel de consommation valide pour la majorité des populations des pays du Nord et une minorité des populations des pays du Sud ;

- l'approfondissement de l'internationalisation financière à travers l'intégration des différentes marchés nationaux en un seul marché financier global (voir 3.).

Le processus d'internationalisation implique une plus grande interdépendance entre les différentes économies nationales et régionales, mais aussi et surtout une hiérarchisation des économies. Suivant un modèle de cercles concentriques ${ }^{2}$, un nombre relativement réduit d'économies correspondant principalement aux pays membres de l'OCDE font partie du noyau dynamique de l'économie mondiale; il faut noter que ce dynamisme s'exprime plus par la vitesse de la création et de la diffusion du progrès technologique que par le niveau du taux de croissance. Dans un second cercle, un certain nombre d'économies, dont les pays du sud-est asiatique et l'un ou l'autre pays latino-américain, participent à ce dynamisme en affichant en particulier des taux de croissance plus élevés que ceux du centre. Dans un troisième et quatrième cercle s'inscrivent la plupart des économies du Tiers-monde, y compris les pays les plus peuplés comme la Chine et l'Inde, qui ne participent que de façon marginale ou sont franchement exclus, telle une bonne partie de l'Afrique, des circuits commerciaux et financiers internationaux. Ainsi aujourd'hui, plus aucune économie, pour vaste et puissante qu'elle soit, ne peut s'isoler des lois du marché international, mais pour autant toutes ne sont pas intégrées de manière dynamique à ce même marché.

2. Le processus d'internationalisation se réalise dans le cadre d'une économie qui reste fondamentalement peu dynamique: le taux de croissance enregistré par les pays de l'OCDE dans les années 80 et celui projeté pour les années 90 reste significativement faible et sensiblement inférieur au taux enregistré dans les années 60 et même dans les années $70^{3}$. On peut considérer que l'économie mondiale est toujours dans la phase récessive d'un cycle long qui aurait commencé au début des années 70. Une des principales caractéristiques de cette phase est la persistance d'un taux de chômage élevé dans la plupart des pays industrialisées, qui est de l'ordre de $10 \%$ en moyenne pour les pays de la 
CE. Ce taux de chômage élevé et apparemment incompressible dans le moyen terme, n'est absolument pas contradictoire avec une reprise des investissements, qui sont soit des investissements de modernisation et de rationalisation dans les secteurs traditionnels, ou qui se réalisent dans les nouveaux secteurs de pointe, mais qui, en tout état de cause, tendent, à court et à moyen terme, à détruire plus d'emploi qu'ils n'en créent. L'accélération du progrès technologique et de sa diffusion au niveau international et la concurrence accrue sur les marchés internationaux qui en découle, entraînent de nouveaux impératifs permanents de restructuration industrielle tant au niveau national qu'international. Les besoins d'investissement dans les secteurs à maturation longue augmentent alors que les critères de rentabilité financière à court terme se font de plus en plus impératifs. Dans un contexte caractérisé par des besoins croissants d'investissements productifs et d'une épargne mondiale qui tend à la baisse ${ }^{4}$, il est difficile de visualiser une réactivation durable de l'économie internationale. Ceci restera vrai tant que l'on n'arrivera pas à définir les nouvelles normes de régulation sociale, en particulier la distribution du revenu, l'organisation du travail et le rôle de l'État, qui permettraient de garantir la rentabilité des investissements productifs qui incorporent les nouvelles technologies.

16 3. La décennie des années 80 aura été marquée par l'hégémonie incontestée du capital financier. La globalisation des marchés financiers correspond surtout à une privatisation et une dérégulation du système financier international qui s'est développé sur une base principalement spéculative. Suivant les dernières estimations, le montant des transactions financières quotidiennes s'élève à environ 1.000 milliards de dollars, soit près de 100 fois le montant des transactions commerciales quotidiennes ou l'équivalent des réserves internationales détenues par les Banques Centrales des pays de l'OCDE ${ }^{5}$. Les mouvements de capitaux internationaux se sont ainsi totalement autonomisés des variables réelles de l'économie mondiale. En outre, ces mouvements se réalisent en dehors de toute forme de régulation et/ou de contrôle de la part d'autorités monétaires nationales ou supranationales.

Cette masse de capitaux flottants à court terme accentue l'instabilité fondamentale d'un système monétaire international, sujet à de fortes variations des taux de change relatifs des principales monnaies et qui ne compte plus avec une véritable monnaie hégémonique. Dans ce système monétaire, les taux de change sont de plus en plus directement liés aux différentiels de taux d'intérêt, et donc aux mouvements de capitaux à court terme. D'autre part, les taux d'intérêts réels particulièrement élevés qui ont prédominé durant toute la décennie reflètent l'hégémonie du capital financier et constituent un obstacle permanent à la mobilisation de ces capitaux dans le secteur productif. La globalisation des marchés financiers, la libéralisation quasi absolue des mouvements de capitaux et la masse critique que représente les capitaux à court terme tendent à accentuer la fragilité et l'instabilité inhérente de tout système financier et augmente la possibilité toujours latente d'une crise majeure à l'échelle internationale. Dans cette perspective, la crise de la dette extérieure latino-américaine, le krach boursier d'août 1987, la crise des caisses hypothécaires des États-Unis, l'effondrement du marché des « junk bonds » en 1990 et la chute, contrôlée de la Bourse japonaise en 1992 devraient constituer pour l'observateur averti autant de rappels du fonctionnement des cycles d'euphorie financière, pour reprendre l'expression de J.K. Galbraith ${ }^{6}$.

17 4. Ces grandes tendances de l'économie mondiale se sont exprimées avec d'autant plus de force, qu'il n'existe pour l'instant ni une puissance hégémonique économique au niveau 
international, ni une instance de coordination supranationale qui puisse les réguler ou en contrôler certains effets pervers.

La suprématie des États-Unis a été mise en brèche dans tous les domaines par ses concurrents directs, en particulier le Japon et la CE; ce déclin se reflète dans les indicateurs suivants :

- perte de participation relative dans le commerce international avec accumulation de déficits commerciaux croissants ;

- restructuration industrielle inachevée et réalisée de manière croissante à travers le recours aux investissements étrangers ${ }^{7}$;

- déclin des grandes banques nord-américaines au profit de leurs concurrentes japonaises (et dernièrement européennes) ;

- financement d'un déficit fiscal apparemment incompressible à travers l'achat renouvelé de Bons du Trésor par des intermédiaires financiers étrangers ;

- perte de la suprématie technologique dans un nombre croissant de secteurs où Japonais (informatique) et Européens (espace, aviation, nucléaire) font des percées qui impliquent la définition des standards internationaux et donc assurent la domination des marchés internationaux pour une période plus ou moins longue ;

- confirmation de la fin du rôle du dollar comme devise-clef de l'économie internationale et remise en question de son statut de monnaie de réserve internationale ;

- incapacité d'imposer les mesures de régulation de l'économie mondiale qui correspondraient à leurs intérêts, par exemple dans les négociations commerciales avec le Japon ou avec l'Europe (agriculture) ou dans la « guerre des taux d'intérêt » avec

l'Allemagne.

Le déclin économique des États-Unis, qui reste une puissance militaire incontestée, n'a pas été compensé par la naissance d'une nouvelle puissance hégémonique et malgré leurs avancées spectaculaires dans certains domaines, ni le Japon ni la CE ne peuvent prétendre jouer pleinement ce rôle à court ou à moyen terme. On trouve une illustration de cette situation tripolaire dans le système monétaire international où coexistent de facto trois zones monétaires correspondant au dollar, au yen et au mark allemand ou par extension au SME. Cependant, les règles du jeu entre ces trois zones ne sont pas encore définies de manière claire et on ne visualise pas un accord qui permettrait de formaliser une institutionnalité internationale reconnue par les différentes puissances régionales.

5. La désintégration à la fin des années $80 \mathrm{du}$ bloc de l'Est et la disparition du « socialisme réel » comme modèle économique a entraîné de nombreuses conséquences sur les plans militaire, politique, économique et idéologique. Sans entrer dans le débat ouvert par cet événement historique, on le considérera seulement sous l'angle d'une nouvelle avancée de l'économie de marché internationale qui intègre ainsi pleinement une partie de l'économie mondiale, qui échappait encore, partiellement et de plus en plus difficilement, à ses lois. Cette expansion de l'économie de marché s'est également vérifiée dans les années 80 à travers l'incorporation d'une partie croissante de secteurs traditionnellement non-marchands (santé, éducation, etc.) et à travers l'approfondissement des relations de marché dans un certain nombre de pays périphériques, plus particulièrement en Amérique latine et en Asie du sud-Est.

Il est trop tôt pour tenter de tirer des conclusions d'ordre général des processus de transition vers l'économie de marché actuellement mis en oeuvre dans les pays d'Europe centrale et de l'ancienne Union soviétique. On peut cependant mentionner que la mort par implosion du « socialisme réel », loin de clore le débat, comme l'affirment certains, 
permet de le recentrer sur les modalités concrètes et les conditions de viabilité du capitalisme comme modèle de développement généralisable à l'ensemble de la planète. La fin de l'utopie socialiste permet de prendre sérieusement en considération des questions telles que la pression du modèle industriel sur l'environnement ou la viabilité d'une distribution internationale du revenu plus inégale que dans n'importe quel pays développé ou sous-développé pris séparément, quand $15 \%$ de la population contrôlent environ $80 \%$ de la richesse mondiale. ${ }^{8}$. Parallèlement, la victoire apparente du néolibéralisme en tant qu'idéologie et sa confrontation avec la réalité induisent une remise en question de l'efficacité économique et sociale du «capitalisme réel $»^{9}$ qui sévit actuellement en Amérique latine et en Europe de l'Est.

6. L'ensemble de ces processus dynamiques se fond dans les deux grandes tendances, plus complémentaires que contradictoires, que de nombreux analystes ont souligné ces derniers temps: la globalisation et la régionalisation de l'économie mondiale. La globalisation se reflète principalement dans la plus grande intégration des différents marchés: de biens, de services financiers et de facteurs de production à l'échelle internationale. On retrouve cette tendance à la globalisation tout autant au sein de chacun de ces marchés qu'entre ceux-ci. Ce processus reflète une nouvelle phase dans la tendance du capitalisme à se constituer en un seul espace économique intégré à l'échelle mondiale.

D'autre part, on constate dans les faits la constitution progressive d'un monde tripolaire sur une base régionale. Les trois zones régionales en voie de formation correspondent aux trois grandes puissances économiques internationales : États-Unis, Japon, CE, associées à leur zone d'influence «naturelle» respective avec laquelle elles entretiennent des liens économiques privilégiés. Ces relations privilégiées sont plus ou moins formalisées et institutionnalisées ou, au contraire, simplement reflet d'une réalité économique. Ainsi, la $\mathrm{CE}$, qui est elle-même un ensemble régional en cours de formation, est associée étroitement aux pays de l'Association Européenne de Libre-Échange (AELE) au sein de l'Espace Économique Européen (EEE) ; elle entretient des relations privilégiées à travers des accords d'association avec les pays d'Europe centrale et certains pays d'Amérique du Nord et, à travers la Convention de Lomé, avec les pays ACP (Afrique, Caraïbes, Pacifique). Par contre, il n'existe aucune forme d'institutionnalisation entre le Japon et les «nouveaux pays industrialisés » de la première et la deuxième génération du Sud-Est asiatique, malgré l'existence de courants commerciaux, financiers et de facteurs productifs importants entre ces pays. Dans le cas des États-Unis, on assiste à un début d'institutionnalisation des relations économiques privilégiées avec le Mexique dans le cadre du NAFTA (North American Free Trade Agreement) et, dans une moindre mesure, avec les autres pays latino-américains à travers les accords-cadre bilatéraux signés dans le contexte de l'Initiative pour les Amériques. Dans ce dernier cas, le processus d'intégration régional se réalise, en fait, plus à travers la dollarisation des économies latinoaméricaines que par le développement des relations commerciales.

20 7. Aux effets souvent déstabilisateurs de ces grandes tendances internationales sur les économies latino-américaines, se sont ajoutés un certain nombre de facteurs internes pour conformer le tableau général de crise qui a caractérisé le développement de la 
région dans les années 80 , qui resteront connues comme la « décennie perdue ». Cette crise généralisée et durable s'est exprimée en particulier par $^{10}$ :

- une perte générale du dynamisme économique qui a frappé tous les secteurs dans à peu près tous les pays de la région. Ainsi pour la première fois depuis les années 30 , la région a enregistré un taux de croissance du PIB par habitant négatif ;

- des déséquilibres macro-économiques persistants malgré l'application systématique de politiques d'ajustement qui cherchaient à les réduire. En particulier, l'inflation s'est accélérée et généralisée jusqu'à déboucher dans des situations d'hyper-inflation dans un certain nombre de pays - entraînant une spirale de dévaluations et hausses des taux d'intérêt. D'autre part, le déficit fiscal s'est révélé particulièrement résistant aux différents traitements qu'on lui a fait subir, l'augmentation continue de la charge de la dette publique extérieure et intérieure annulant les effets des réductions successives des dépenses publiques;

- une détérioration de la situation sociale, dérivée du caractère régressif de l'ajustement, qui s'est exprimée à travers une forte diminution du salaire réel, une explosion du secteur informel urbain et rural, une augmentation de la précarité de l'emploi et des revenus et une forte accumulation de la « dette sociale»;

- un affaiblissement du secteur public en général et une redéfinition du rôle de l'État dans le développement à travers, entre autres, une vague de privatisations et la réduction de l'autonomie de l'État dans la définition et l'application des politiques économiques ;

- une augmentation (jusque 1987) de la dette extérieure malgré des transferts extérieurs nets représentant environ $4 \%$ du PIB au niveau régional ;

- une chute drastique du taux d'investissement, d'environ $23 \%$ du PIB dans les années 70 à $16 \%$ à partir de 1982, ce malgré un maintien du taux d'épargne national autour de 22 à $24 \%$ du PIB. Cette réduction du taux d'investissement compromet sérieusement à la fois le succès des politiques de modernisation de l'appareil productif menées à bien dans certains pays et le développement futur de la région en général.

\section{Les relations économiques entre l'Amérique latine et la Communauté européenne dans les années 80}

21 Les relations économiques actuelles entre l'Amérique latine et les pays membres de la Communauté européenne sont le produit de l'évolution historique de ces mêmes relations, du contexte international dans lequel elles se réalisent et de l'évolution interne de chacun des partenaires. Dans les années 80 , cette dernière se caractérise pour l'Amérique latine par la crise de la dette extérieure et les politiques d'ajustement qui visent entre autres à établir de nouvelles modalités d'insertion internationale; pour l'Europe, on retiendra principalement, les effets dérivés de la construction de l'intégration communautaire et la restructuration économique, induite par l'incorporation massive du progrès technologique. Dans l'évaluation de ces relations, il faut prendre en compte le fait que sur le plan économique, l'Amérique latine constitue une des toutes dernières priorités dans l'agenda européen. En effet, la CE est d'abord préoccupée de sa propre construction, en particulier de l'approfondissement du processus d'intégration à travers la préparation du Marché unique qui entrera en vigueur en 1993 et l'implantation des politiques économiques de convergence qui doivent rendre possible la création de l'Union Économique et Monétaire d'ici à la fin du siècle. Son second champ de priorité se réfère à son élargissement futur, privilégiant pour cela les 
relations avec les pays membres de l'Association Européenne de Libre Échange (AELE), qui formeront avec elle l'Espace Économique Européen, et avec les pays d'Europe centrale susceptibles d'entrer à moyen terme dans la Communauté. La troisième priorité se réfère à ses principaux partenaires et concurrents économiques, soit les États-Unis et le Japon. En quatrième lieu, la Communauté est liée, à travers des accords d'association, avec la plupart des pays de la Méditerranée Sud et, dans le cadre de la Convention de Lomé, avec les pays ACP. Malgré des relations historiques privilégiées et un certain rapprochement sur le plan politique, l'Amérique latine tend à se situer dans le bas des priorités économiques de la CE. Cette tendance semble s'être confirmée dans les années 80, bien qu'on puisse noter dans les dernières années un regain d'intérêt de la part des Européens qui, espère-t-on, n'est pas uniquement lié à un éphémère anniversaire du cinquième centenaire de la première « découverte » de l'Amérique latine.

1. Les relations commerciales entre les deux régions se caractérisent par une marginalisation réciproque croissante et une structure asymétrique de leurs échanges.

La participation de l'Amérique latine dans les importations et les exportations extracommunautaires de la CE n'a cessé de diminuer depuis 1965 au point de ne plus représenter en 1990 que $5,5 \%$ des importations et 3,8\% des exportations extracommunautaires de la CE. Parallèlement, la CE représente également une proportion décroissante des importations et des exportations de l'Amérique latine, quoique dans une moindre mesure. Cette tendance à long terme s'est accentuée durant la dernière décennie : l'Amérique latine est la région qui a le plus perdu de parts relatives dans le marché communautaire et les exportations de la Communauté vers l'Amérique latine ont diminué fortement et durablement en termes absolus et n'ont retrouvé qu'en 1990 le niveau qu'elles avaient atteint en 1980.

Tableau 1 : Les relations commerciales CE - Amérique latine 1980 -1990 (millions de USD)

\begin{tabular}{|l|l|l|}
\hline & Importations $\mathrm{CE}^{1}$ & Exportations $\mathrm{CE}^{2}$ \\
\hline 1980 & 23.160 & 19.086 \\
\hline \hline 1985 & 23.104 & 11.872 \\
\hline 1990 & 32.704 & 21.540 \\
\hline
\end{tabular}

1. Importation de la CE en provenance de l'Amérique latine

2. Exportations de la CE vers l'Amérique latine

Source : IRELA, sur base de données du FMI et de EUROSTAT

L'asymétrie de ces relations commerciales a une double dimension. D'abord l'Amérique latine constitue un marché et un fournisseur marginal et facilement substituable pour la CE. Au contraire, la CE représente toujours le second marché pour les exportations latinoaméricaines et est son second fournisseur. Pour un certain nombre de pays, parmi lesquels se trouvent certains des plus importants, la CE reste toujours leur premier marché et/ou leur premier fournisseur. 
Tableau 2 : Participation relative de la CE et de l'Amérique latine dans leur commerce international 1980-1990 (\%)

\begin{tabular}{|l|l|l|l|l|}
\hline & Part CE dans & & \multicolumn{2}{l|}{ Part Amérique latine dans } \\
\hline & M - AL & $\mathrm{X}-\mathrm{AL}$ & $\mathrm{M}-\mathrm{CE}^{1}$ & $\mathrm{X}-\mathrm{CE}^{1}$ \\
\hline 1980 & 19.1 & 23.8 & 5.9 & 6.4 \\
\hline 1958 & 17.4 & 22.9 & 7.5 & 4.1 \\
\hline 1990 & 18.4 & 23.8 & 5.5 & 3.8 \\
\hline
\end{tabular}

1. Importations et exportations extracommunautaires

Source : IRELA, sur base de données FMI et EUROSTAT

Mais, c'est surtout au niveau de la structure des produits exportés par les deux régions que cette asymétrie est manifeste. En effet, environ $80 \%$ des exportations latinoaméricaines vers la $\mathrm{CE}$ sont constituées de produits primaires (agricoles, miniers et pétrole), tandis qu'environ $90 \%$ des exportations communautaires vers l'Amérique latine sont constituées de produits manufacturés dont près de $50 \%$ correspondent à des biens de capital, de l'équipement de transport et des produits chimiques. En outre, même si la structure d'exportation latino-américaine peut paraître relativement diversifiée, les 5 principaux produits d'exportation, qui signifient chacun plus de $5 \%$ des exportations totales, représentent près de $40 \%$ du total, tandis qu'aucune rubrique d'exportation de la Communauté ne dépasse plus de $5 \%$ du total et que les 5 premières rubriques représentent seulement $16 \%$ du total ${ }^{11}$.

Bien que tous les pays des deux régions sont impliqués dans les échanges commerciaux, la plus grande partie de ceux-ci se concentrent en fait entre un nombre limité de partenaires. Ainsi, le Brésil, l'Argentine, le Mexique et le Chili représentent $70 \%$ des importations en provenance de la CE et les mêmes pays plus le Venezuela, $76 \%$ des exportations vers la CE, tandis que l'Allemagne, l'Italie, la France et la Grande-Bretagne absorbent $76 \%$ des exportations latino-américaines et avec la Hollande, signifient $75 \%$ des importations latino-américaines provenant de la $\mathrm{CE}^{12}$.

Enfin, il faut signaler que les exportations latino-américaines ne bénéficient d'aucun régime préférentiel de la part de la $\mathrm{CE}$, sauf le Système de Préférences Généralisées (SPG) qui ne profite qu'à environ $10 \%$ des exportations latino-américaines. Au contraire, $28 \%$ des exportations latino-américaines ont dû affronter d'une manière ou d'une autre des barrières non-tarifaires, relevant des pratiques néoprotectionnistes employées systématiquement par la CE, tout comme d'ailleurs par les autres pays industrialisés. Il est encore trop tôt pour pouvoir mesurer l'impact de la franchise tarifaire accordée par la Communauté aux pays andins (Bolivie, Colombie, Équateur, Pérou) pour la période 1991-1994, dans le cadre de la politique internationale, visant à décourager la culture de la coca et le trafic de la cocaïne.

27 C'est dans le domaine des relations commerciales que l'on trouve le plus grand nombre de conflits d'intérêts entre les deux régions. En particulier dans le commerce des produits agricoles, différents pays des deux régions sont exportateurs nets de produits tempérés 
(blé, oléagineux, viande) et se livrent à une âpre concurrence sur les marchés mondiaux, d'autant plus que le marché européen est largement protégé à travers les mécanismes mis en place dans le cadre de la Politique Agricole Commune (PAC). Les principaux produits tropicaux exportés par l'Amérique latine sont en concurrence avec les exportations des pays ACP qui bénéficient d'un accès préférentiel au marché communautaire. En outre, certains produits tropicaux doivent affronter directement la concurrence européenne; ainsi, le sucre peut être produit aussi bien en agriculture tempérée qu'en agriculture tropicale, et bien que cela puisse paraître paradoxal, la CE est aussi un important producteur de bananes. Les producteurs européens (Grèce, Madère, Açores et Antilles françaises) jouissent de la préférence communautaire et bien qu'extrêmement compétitifs, les exportateurs latino-américains n'ont accès qu'aux marchés des pays qui ne sont pas producteurs ou n'ont pas d'accords privilégiés avec certains exportateurs (France, Angleterre) ${ }^{13}$, soit les marchés allemand, danois et du Benelux.

Un second point d'intérêt majeur pour l'Amérique latine, surtout à un moment où elle tente de moderniser et diversifier la structure de ses exportations, a trait à l'accès au marché communautaire pour ses exportations de produits manufacturés. Celles-ci rencontrent encore trop souvent de nombreux obstacles sous la forme de barrières nontarifaires (BNT) sans cesse plus sophistiquées, qui vont depuis les traditionnels contingentements et "accords volontaires » de restriction à l'exportation jusqu'aux mesures soi-disant liées à la protection de l'environnement.

2. Les relations financières se caractérisent par une participation importante des grandes banques privées d'origine européenne dans le processus d'endettement latino-américain. Ainsi, fin 89, la dette extérieure latino-américaine avec les banques commerciales européennes ${ }^{14}$ s'élevait à 81.6 milliards de dollars, contre des encours de 58.9 milliards avec les banques nord-américaines ${ }^{15}$. Cette situation se retrouve dans le cas de tous les grands débiteurs latino-américains, à l'exception du Mexique.

Cette situation, généralement méconnue, a influencé le déroulement du processus de renégociation et plus récemment de réduction de la dette. En effet, dans un premier temps, la plupart des analystes ont eu tendance à considérer qu'il s'agissait fondamentalement d'un problème entre les gouvernements latino-américains et les grandes banques nord-américaines. En effet, le système bancaire nord-américain reste de loin le plus gros créancier de l'Amérique latine, si l'on considère les pays européens séparément; d'autre part, les grandes banques («money centers») nord-américaines étaient largement plus engagées et plus exposées que leurs concurrentes européennes. Dans la première phase de renégociation, caractérisée par le rééchelonnement des dettes, ce sont effectivement les grandes banques américaines qui ont dirigé les syndicats bancaires chargés de cette négociation ${ }^{16}$.

Dans les deuxième et troisième phases de la gestion de la crise de la dette, caractérisée par le développement des opérations de swap sur le marché secondaire et par la réduction négociée de la dette dans le cadre du plan Brady, les banques européennes ont manifesté des intérêts de plus en plus divergents qui s'exprimeront dans des stratégies différentes de celles suivies par les banques nord-américaines. En particulier, sur la base d'un traitement fiscal différencié des provisions pour crédits douteux, les banques européennes sont moins favorables à la réduction de la valeur nominale de la dette envisagée par le plan Brady, et encore moins disposées à apporter des capitaux frais. Dans les différents accords négociés jusqu'à présent avec les pays débiteurs, elles ont donc 
généralement opté pour une réduction des taux d'intérêt qui ne remet pas en cause la valeur comptable de la dette ${ }^{17}$.

On constate ainsi une fracture dans le cartel bancaire international qui pourrait être exploité pour obtenir de meilleures conditions de renégociation pour les pays débiteurs, mais aussi une plus grande rigidité de la part des créanciers d'origine européenne.

3. L'investissement étranger direct d'origine communautaire a suivi durant les années 80 le comportement général de l'investissement étranger direct total en Amérique latine, soit une rétraction à partir de 1982 et une récupération dans les dernières années de la décennie.

Bien que les États-Unis restent la première source d'investissements étrangers directs en termes de stocks accumulés pour l'ensemble du sous-continent latino-américain et pour la plupart de ces pays avec l'exception notable du Brésil (où les investissements des 12 dépassent légèrement ceux des États-Unis ${ }^{18}$, dans les dernières années, les investissements en provenance des pays de la Communauté sont substantiellement supérieurs à ceux d'origine nord-américaine pour la région prise dans son ensemble ${ }^{19}$. On notera en particulier une croissance accélérée des investissements d'origine européenne au Mexique, investissements réalisés par des firmes qui cherchent à se positionner favorablement dans le cadre de la mise en place du NAFTA ${ }^{20}$. D'autre part, on remarquera une participation active des entreprises transnationales d'origine européenne dans le processus de privatisation des entreprises publiques latino-américaines, particulièrement au Mexique. en Argentine, au Chili et au Venezuela. Enfin, il est intéressant de noter que, dans certains domaines comme les télécommunications et l'aviation, le processus de privatisation se réduit en fait à une opération de rachat des entreprises publiques latinoaméricaines par des entreprises parastatales européennes. Le cas le plus notable est certainement celui des parastatales espagnoles, Iberia et Telefonica, qui, à travers leur politique de rachat systématique des entreprises latino-américaines privatisées dans leur domaine respectif. préparent le terrain pour la phase tardive d'internationalisation du capital privé espagnol dans cette région.

31 4. La CE est le premier partenaire de l'Amérique latine dans le domaine de l'Assistance officielle au développement (AOD), loin devant les États-Unis, si l'on exclut les programmes de coopération militaire. Les ressources allouées à l'Amérique latine par les pays membres de la CE ont plus que doublé entre 1985 et 1989, passant de 480 millions à 1,180 millions de dollars. A l'aide bilatérale octroyée par les pays membres, il faut ajouter l'aide multilatérale gérée directement par la Commission des Communautés européennes ; pour la période 1991-1995, celle-ci s'élève à environ 1 milliard d'ECU, soit l'équivalent de 250 millions de dollars annuels contre 140 millions de dollars annuels pour la période 1985-1990. L'assistance officielle au développement bénéficie principalement aux pays les plus pauvres de la région et/ou aux groupes les plus défavorisés de la population de ces pays. La Commission de la CE a adopté récemment les nouvelles orientations relatives aux priorités de l'assistance communautarisée, c'est-à-dire gérée directement par la Commission.

Ces nouvelles orientations privilégient l'assistance au développement rural au sens large, y compris la lutte contre la drogue et la protection de l'environnement, ainsi que la dimension humaine du développement c'est-à-dire la prise en compte des problèmes spécifiques de groupes sociaux comme les groupes ethniques autochtones ou le rôle des femmes dans le développement ${ }^{21}$. 
5. Un des domaines le plus prometteur pour une réactivation durable des liens économiques entre la Communauté et l'Amérique latine est probablement celui de l'intégration économique régionale. En effet, les deux régions sont celles qui ont le plus d'expérience et qui ont le plus avancé dans la construction d'ensembles économiques régionaux ou sub-régionaux.

Certes, il faut prendre en compte le fait que le processus d'intégration européen est qualitativement différent dans le sens où la création d'une zone de libre-échange commerciale et ensuite d'un marché unique intégré ont été dès l'origine considérés non comme des fins en soi, mais comme des étapes nécessaires vers l'objectif plus ambitieux qu'est l'intégration politique. Celle-ci, à son tour, a été conçue comme le moyen idoine pour maintenir de façon durable la paix en Europe. Au contraire, les processus d'intégration régionale ou sub-régionale latino-américain visent principalement à la création d'une zone de libre commerce et, dans certains cas (MCCA, Mercosur), d'un marché commun pouvant impliquer la libre circulation des facteurs de production. De même, le NAFTA ne poursuit d'autres ambitions que la consolidation d'une zone de libreéchange commerciale, qui permettrait, en outre, la libre circulation du capital, tout en restreignant celle de la main-d'oeuvre.

Les expériences tant européennes que latino-américaines ont démontré que le développement des zones de libre-échange régional ne sont en rien incompatibles avec la croissance du commerce international avec les partenaires extra-régionaux ${ }^{22}$. On a au contraire enregistré des augmentations tant du commerce intra-régional que du commerce extra-régional dans les deux cas. Dès lors, l'approfondissement de l'intégration en Europe et la réactivation des différents schémas subrégionaux en Amérique latine ne sont pas à priori un obstacle à la relance du commerce entre les deux régions.

Selon les résultats d'une étude récente réalisée par l'IRELA ${ }^{23}$, l'impact global de la réalisation du Marché unique européen en 1993 sur le développement de l'Amérique latine devrait être relativement marginal et ses conséquences sur les échanges entre les deux régions seraient relativement moins importantes que pour d'autres régions plus étroitement liées à la CE.

La construction parallèle de l'intégration économique régionale dans les deux régions peut servir de base à une meilleure compréhension réciproque des stratégies de développement respectives, à travers la confrontation des expériences. Elle assure ainsi une base objective à une réactivation des flux d'investissements et des flux financiers, ainsi qu'à la nécessaire restructuration des échanges commerciaux.

\section{L'agenda pour les années 90}

De l'analyse qui précède se détachent certaines convergences et autant de divergences dans les relations économiques entre l'Amérique latine et la Communauté européenne. L'importance relative de ces points, les moyens de tirer parti des convergences et les solutions à apporter aux conflits d'intérêts réels ou potentiels, sont appréciés de manière souvent différente par les deux partenaires. Le premier obstacle à une réactivation durable de leurs relations réside donc dans la définition même de l'agenda des négociations. La présentation qui suit de cet agenda ne cherche en rien à refléter les conceptions de l'une ou l'autre région et ne prétend être ni exhaustive, ni strictement objective. 

compliquées que, des deux côtés, on a tendance à déplorer la confusion relative à l'identification des instances avec lesquelles traiter les différents problèmes. Ceci reflète, par ailleurs, une relative méconnaissance réciproque des institutions respectives. Ainsi, l'Europe regrette souvent l'absence d'institutions régionales latino-américaines qui pourraient discuter d'égal à égal avec la Commission de la CE. De leur côté, les négociateurs latino-américains trouvent parfois difficile de s'y retrouver dans la répartition des compétences entre les différents organes communautaires (Commission, Conseil, Parlement, Banque Européenne d'Investissement, etc.) et entre ces organes et les pays membres.

points les plus délicats de l'agenda se rapportent à l'aménagement des relations commerciales entre les deux régions et correspondent, de fait, à une demande répétée de la partie latino-américaine, souvent éludée par les interlocuteurs européens. Il convient ici de séparer les revendications " traditionnelles ", relatives au protectionnisme agricole européen et à l'extension du SPG, de celles qui tendent à occuper récemment le devant de la scène, relatives à l'accès au marché communautaire des exportations latinoaméricaines dans des conditions « de marché », c'est-à-dire ni préférentielles, ni soumises à des barrières non tarifaires (BNT) spécifiques. En ce sens, l'Amérique latine, et en particulier les pays les plus avancés, demande seulement la reconnaissance de son statut de partenaire commercial à part entière, et non plus de sujet mineur à qui l'on accorde un traitement « préférentiel » sous différentes modalités.

Malgré leur importance, les revendications «traditionnelles" sont d'autant moins susceptibles d'être considérées favorablement par la CE qu'elles ont des implications qui débordent largement de la sphère de ses relations commerciales avec l'Amérique latine. Ainsi, l'extension de la couverture du SPG s'appliquerait, par définition, à tous les autres pays qui en bénéficient ; elle susciterait également des réactions négatives des pays ACP qui verraient réduit le caractère préférentiel de leurs relations commerciales avec la $\mathrm{CE}$.

Comme on l'a déjà mentionné, une partie substantielle des exportations agricoles latinoaméricaines sont affectées directement et indirectement, par les modalités d'application de la PAC, en particulier le principe de la "préférence communautaire » et les prix garantis, qui se traduisent en subventions à l'exportation, les termes de la réforme récente de la PAC, résultats d'un laborieux compromis entre les Douze, et le blocage des négociations sur le commerce des produits agricoles entre la CE et les États-Unis dans le cadre de l'Uruguay Round sont de claires indications des limites des concessions que la CE est disposée à faire dans le réaménagement de sa politique agricole. Celle-ci constitue un des axes fondamentaux de la construction européenne et ses principes de base ne sont manifestement pas sujets à négociation avec des partenaires extérieurs. On notera que les subventions à la production et à la consommation des produits agricoles pratiquées par la $\mathrm{CE}$ correspondent à la moyenne de celles appliquées par les pays de l'OCDE, légèrement supérieures à celles des États-Unis, mais largement inférieures aux niveaux atteints par le Japon ou les pays scandinaves.

Tableau 3 : Les aides gouvernementales à l'agriculture dans les pays de l'OCDE

Équivalents subventions à la production et à la consommation par pays 


\begin{tabular}{|l|l|l|l|}
\hline & \multicolumn{2}{l|}{$\begin{array}{l}\text { ESP } \\
\text { (\% valeur de la } \\
\text { consommation) }\end{array}$} & $\begin{array}{l}\text { ESP } \\
\text { (\% valeur de la consommation) }\end{array}$ \\
\hline & & & \\
\hline Australie & $1979-1985$ & 1989 & 1989 \\
\hline Autriche & 29 & 10 & 7 \\
\hline Canada & 30 & 35 & 23 \\
\hline CEE & 35 & 38 & 31 \\
\hline États-Unis & 26 & 27 & 15 \\
\hline \hline Finlande & 57 & 72 & 68 \\
\hline Japon & 64 & 72 & 48 \\
\hline \hline Norvège & 71 & 74 & 58 \\
\hline Nouvelle-Zélande & 23 & 5 & 5 \\
\hline \hline Suède & 67 & 47 & 49 \\
\hline Suisse & 34 & 39 & 31 \\
\hline Moyenne OCDE & 75 & 53 \\
\hline
\end{tabular}

Source : chiffres OCDE, cités par J.F. ECK. Le commerce mondial des produits agricoles au XXème siècle, Ed. Eyrolles, Paris 1992, p. 191.

Les seules négociations susceptibles d'aboutir dans ce domaine seraient celles portant sur des produits spécifiques, de particulière relevance pour les pays exportateurs et plutôt marginales pour la $\mathrm{CE}$, et qui ne remettent pas en cause les principes de base de la PAC. Ainsi, dans le cas des exportations latino-américaines de bananes, on ne peut guère attaquer la "préférence communautaire» par laquelle la $\mathrm{CE}$ protège les producteurs « européens », malgré leur manque de compétitivité, mais bien insister sur le fait que le maintien du contingentement national du marché communautaire va à l'encontre d'un autre principe de base du Marché unique, celui de la libre circulation des marchandises et implique un manque à gagner pour le consommateur européen et les producteurs extracommunautaires.

Les conditions d'accès des exportations latino-américaines de produits manufacturés au marché communautaire devraient constituer le coeur des négociations commerciales entre les deux régions. Suivant les principes de la «transformation productive ", nouvelle priorité de l'Amérique latine ${ }^{24}$, celles-ci devraient constituer le moteur de la nouvelle insertion de la région dans l'économie mondiale. Les demandes de garantie d'accès au 
marché sont d'autant plus légitimes que s'est déjà réalisée l'ouverture unilatérale des marchés latino-américains dans le cadre des politiques de libéralisation mises en place par la plupart de ces pays (cf. tableau 4). Or, la CE a procédé, dans les années 80 , à l'implantation d'un nombre croissant de BNT, toujours plus sophistiquées, telles les mesures compensatoires et anti-dumping. L'utilisation des BNT est d'autant plus préjudiciable pour les exportateurs, que leur application intervient généralement lorsque ceux-ci arrivent à pénétrer de manière significative, et donc potentiellement durable, un créneau du marché. Leur caractère discriminatoire et arbitraire tend à biaiser la dynamique exportatrice et accentue l'incertitude de l'investissement dans ces secteurs. En outre, l'utilisation des BNT pour protéger des secteurs industriels en difficulté ou en phase de restructuration va directement à l'encontre des principes qui sous-tendent la construction du Marché unique et des objectifs de concurrence et de compétitivité internationales qu'il poursuit. On ne peut d'un côté prôner dans les forums internationaux des politiques d'ajustement visant à favoriser les exportations et, de l'autre, fermer son propre marché à ces exportations.

Tableau 4 : Évolution des tarifs douaniers en Amérique latine

\begin{tabular}{|c|c|c|c|c|}
\hline & \multicolumn{2}{|c|}{ Tarif maximum (\%) } & \multicolumn{2}{|c|}{ Tarif moyen (\%) } \\
\hline & antérieur ${ }^{1}$ & actuel $^{2}$ & antérieur ${ }^{1}$ & actuel $^{2}$ \\
\hline Argentine & 53 & 22 & 29 & 10 \\
\hline Brésil & 105 & 85 & 38 & 25 \\
\hline Chili & 35 & 11 & 35 & 11 \\
\hline Colombie & 100 & 50 & 46 & 34 \\
\hline Mexique & 100 & 20 & 29 & 13 \\
\hline Pérou & 110 & 50 & 76 & 32 \\
\hline Uruguay & - & 40 & - & 28 \\
\hline
\end{tabular}

1. En vigueur avant l'application de la réforme commerciale

2. 1991

Source : F. PORTA, Apertura comercial e integracion en America Latina, SELA, Caracas, juillet 1991.

Dans ce sens, la réactivation durable et la restructuration des courants commerciaux entre les deux régions dépend, en bonne partie, de l'application par l'Europe d'une politique commerciale conforme à ses principes et à ses intérêts à long terme.

2. Le deuxième point de l'agenda se référerait, selon nous, au problème de la dette extérieure qui est loin d'être résolu et qui constitue toujours une sérieuse entrave à la réactivation économique de l'Amérique latine. Avant tout, il faut souligner que cette question n'entre en rien dans les compétences des instances communautaires 
européennes, contrairement à la politique commerciale qui est décidée par le Conseil sur proposition de la Commission.

Ces dernières années, on a enregistré de sérieux progrès dans le traitement de la dette et de son service, en particulier à travers les réductions négociées du principal et/ou des intérêts dans le cadre du plan Brady, entre les banques commerciales et la plupart des grands débiteurs latino-américains (Mexique, Venezuela, Argentine, ...). En outre, un grand nombre de petits pays, parmi lesquels les plus pauvres de la région, ont bénéficié de la condonation partielle de leur dette publique par les États-Unis et certains pays européens. Surtout depuis 1990, on assiste à un afflux massif de capitaux vers la région - 18.4 milliards de dollars en 1990 et 36 milliards de dollars en 1991 - qui se sont traduits en 1991 par un transfert de ressources extérieures net positif pour la région pour la première fois depuis la crise de $1982^{25}$.

Cependant, on n'a toujours pas apporté de solution durable à la question de la dette. Malgré la baisse des taux d'intérêts internationaux et les réductions négociées, les paiements de l'Amérique latine, au titre d'intérêts, représente toujours environ 30 milliards de dollars annuels, soit environ $3 \%$ du PIB. Les mouvements de capitaux mentionnés sont concentrés dans quatre pays : Mexique (19.5 milliards en 91), Argentine, Chili et Venezuela. Une grande partie de ces capitaux se sont investis dans les Bourses locales et restent donc extrêmement volatiles et n'ont pas entraîné jusqu'à présent une relance de l'investissement productif. Enfin, la majeure partie des pays se trouvent toujours dans des situations de suspension partielle du paiement du service de la dette, souvent bien malgré eux, et les arriérés d'intérêts de la région s'élèvent à environ 25 milliards de dollars ${ }^{26}$.

Face à cette situation et au vu de l'importance des créances des banques commerciales européennes, il parait souhaitable que l'Europe adopte une position commune qui favorise une négociation de fond sur la dette extérieure, de façon à ce que les ressources utilisées pour le service de la dette puissent être mobilisées pour le développement économique de la région.

3. Dans le cadre des politiques d'ajustement structurelles, la plupart des pays d'Amérique latine ont mis en oeuvre un processus radical d'ouverture, commerciale et financière, de leurs économies et ont mis en place une série de dispositions favorisant les investissements étrangers, entre autres à travers la libéralisation des dispositions les concernant et à travers le processus de privatisation. L'Amérique latine est en droit d'attendre une réponse positive des investisseurs internationaux dans des termes qui soutiennent le processus de réactivation et de modernisation de l'appareil productif. En d'autres termes, l'investissement étranger doit apporter avec lui de réels transferts de technologie et des mécanismes d'accès aux marchés internationaux. Dans ce sens, on rappellera que le rachat d'une entreprise, publique ou privée, n'implique pas en soi un investissement net, à moins qu'il ne s'accompagne d'un compromis de capitalisation et de modernisation de l'entreprise concernée. De même, les investissements en Bourse, essentiellement spéculatifs, ne se traduisent pas nécessairement par des investissements productifs.

Même si les décisions d'investissement restent fondamentalement de la compétence des agents économiques privés, les instances de décision publique, étatales ou communautaires, ont un rôle important à jouer dans la création des conditions favorisant ces décisions. Ainsi, par exemple, même s'il reste relativement marginal, l'accès aux fonds de la Banque Européenne d'Investissement pourrait stimuler la concrétisation de projets 
d'investissement, entre autres sous forme de joint-ventures, qui répondent aux critères de transfert technologiques, de formation du capital humain et d'exportations souhaités par les pays récepteurs.

Parallèlement, l'association des chercheurs des pays latino-américains qui l'ont sollicité aux programmes de recherche communautaires, tels EUREKA ou ESPRIT, constitue un mécanisme idoine pour appuyer le processus de modernisation en Amérique latine, stimuler le transfert technologique et la formation scientifique et, à long terme, ouvrir de nouveaux marchés pour les produits issus de ces programmes de recherche.

4. Dans les préoccupations latino-américaines s'est inscrite récemment la «concurrence » des pays d'Europe centrale, tant sur le plan de l'accès au marché que de la captation des ressources financières sous forme d'investissements, de prêts ou d'aide au développement. Jusqu'à présent, on a pu constater que les faits n'ont pas confirmé ces attentes négatives. Les ressources financières privées se sont dirigées plutôt vers l'Amérique latine que vers l'Europe de l'Est. Au niveau communautaire, le Parlement Européen est parvenu à imposer aux autres instances sa conception suivant laquelle l'aide publique à la reconstruction de l'Europe Centrale ne devait pas se faire au dépens de l'aide au développement des pays du Sud. Sur le plan commercial, il paraît évident que les pays d'Europe centrale représentent de sérieux concurrents dans un certain nombre de branches industrielles pour les exportateurs latino-américains sur le marché communautaire, d'autant plus qu'ils sont favorisés par les accords d'association signés par la CE. Cependant, dans un certain nombre de domaines, ces économies sont plus complémentaires que concurrentes des économies latino-américaines; aussi, à mesure qu'elles se reconstruisent et que le niveau de consommation de leurs populations augmente, elles constituent des marchés potentiellement dynamiques pour certaines exportations latino-américaines. Il appartient aux entreprises latino-américaines de se positionner dès maintenant sur ces marchés afin de bénéficier de leur dynamisme futur.

5. Last but not least, la protection de l'environnement se convertira probablement en un des thèmes majeurs de l'agenda des relations europeo-Iatino-américaines dans les années 90. Hissée au rang des toutes premières priorités des politiques communautaires, tant sur le plan interne qu'au niveau international, cette question correspond à des situations d'extrême urgence en Amérique latine. Le degré de pollution de villes comme Mexico ou Santiago, qui oblige aux moments les plus critiques la paralysation des activités économiques, ou la déforestation massive de l'Amazonie, au Brésil mais aussi en Colombie, et le processus de désertification qui en découle, sont seulement les exemples les plus frappants de la dégradation de l'environnement qui touche la région ${ }^{27}$

La dimension mondiale de la plupart des problèmes écologiques les convertit en une sphère privilégiée de la coopération internationale, où peuvent se rencontrer les intérêts objectifs de toutes les parties impliquées. On ne peut, cependant écarter à priori l'expression de sérieuses divergences relatives à la définition de la responsabilité des différents agents économiques et à la répartition entre eux des coûts qu'entraînera nécessairement une meilleure protection de l'environnement. Des tensions pourraient aussi se manifester dans des domaines tels que le strict respect des souverainetés nationales ou l'utilisation de critères écologiques comme mécanismes néoprotectionnistes déguisés. 


\section{Conclusion}

41 La réactivation des relations économiques entre l'Amérique latine et la Communauté européenne dans les prochaines années dépendra des grandes tendances de l'économie internationales et des stratégies de développement suivies par chacune des régions, mais aussi de leur capacité, et donc de leur volonté politique, de mettre en valeur leurs intérêts communs, tout en reconnaissant leur identité propre et en assumant leurs différences.

1. L'économie mondiale devrait toujours se caractériser par l'accélération de l'internationalisation dans toutes ses dimensions, productive, technologique et financière, l'approfondissement et l'expansion de l'économie de marché, la création et la diffusion accélérée du progrès technologique dans un cadre de faible croissance. La combinaison de ces tendances devrait se traduire par une accentuation des processus de restructuration et de re-hiérarchisation des économies nationales et régionales. A faute d'émergence d'une nouvelle puissance hégémonique ou de mécanismes de coordination supranationaux, la caractéristique fondamentale pourrait bien être l'instabilité, accrue par les tensions accumulées dans un système financier et monétaire international plus sensible que jamais.

2. La Communauté européenne affronte des défis historiques pour elle-même et le reste $\mathrm{du}$ monde. Son approfondissement et sa consolidation passent par la réalisation du Marché Unique, largement acquise, et de l'Union économique et monétaire, actuellement en cours. Comme l'ont montré, a contrario, les remous monétaires de septembre 1992, la création d'une monnaie unique qui puisse assumer le rôle de monnaie de réserve internationale, constitue une urgence tant pour la Communauté que pour introduire un facteur de stabilisation dans le système monétaire international. L'élargissement de la $\mathrm{CE}$ et la reconstruction des économies de l'Europe centrale permettront de consolider un pôle de stabilité dans un monde en cours de restructuration. Seulement une Europe consolidée et élargie pourra affronter des défis tels que les conséquences de la désintégration de l'ex-Union Soviétique ou le nécessaire réaménagement des relations Nord-sud.

44 3. L'Amérique latine s'est engagée, à des degrés divers selon les pays, dans un processus de réformes substantielles de son économie pour s'adapter aux exigences de la nouvelle donne internationale et tenter de renouer avec le développement économique. Certains succès prometteurs obtenus jusqu'ici restent cependant limités et, surtout, instables dans le temps. Aux politiques de libéralisation et aux efforts consentis pour renforcer la "compétitivité nationale» (au sens de la CEPAL) à travers la modernisation et l'ouverture, doivent correspondre une garantie d'accès aux marchés des pays industrialisés - c'est ce que le Mexique a négocié dans le cadre du NAFTA - et une relance de l'intégration régionale, actuellement en cours. Une réactivation durable de l'économie dépend, non seulement de la restauration et du maintien des grands équilibres macroéconomiques, mais surtout de la relance des investissements productifs qui tendent à rester à des niveaux significativement bas, même dans les pays qui ont bénéficié récemment d'afflux massifs de capitaux. La réinsertion dynamique de la région dans l'économie mondiale dépend, certes de ses propres efforts, mais aussi de la prise en considération par ses principaux partenaires économiques des réaménagements nécessaires dans les domaines de l'accès aux marchés, des transferts réels de technologie 
et des modalités des mouvements de capitaux, en privilégiant l'investissement productif, et en acceptant, enfin, une véritable solution au problème de la dette extérieure.

4. Dans ce contexte, les deux régions profiteront mutuellement de la consolidation et la stabilisation de leurs stratégies respectives de développement. Elles le feront d'autant plus que leurs autorités respectives prennent conscience des conflits d'intérêt transitoires que leur mise en oeuvre peut entraîner et qu'elles aient la volonté politique de les surmonter tout en prenant en compte les intérêts fondamentaux de chacun. Ceci implique mettre dans l'agenda des négociations économiques les véritables problèmes énoncés par chacune des parties, pour déterminer les points d'accord possibles et accepter que certains accords soient impossibles pour l'instant. Les relations économiques entre les deux régions se sont clairement revitalisées durant les dernières années ; pour leur donner un caractère durable, il s'agit maintenant de discuter en termes clairs des obstacles que leur propre dynamisme peut entraîner, comme des problèmes de fonds qui ont trop longtemps marginalisé ces relations. 1992 aura, au moins, eu pour mérite d'avoir suscité une "redécouverte " mutuelle. Cependant les grandes tendances sous-jacentes de l'économie mondiale, telles l'internationalisation et la régionalisation n'impliquent pas a priori une réactivation durable, ni une restructuration de ces relations. Sans doute, la concrétisation de celles-ci exigera-t-elle une bonne dose de "volontarisme ", sous la forme de nouvelles modalités de coopération économique entre partenaires commerciaux, qui puissent mener les discussions d'égal à égal.

\section{BIBLIOGRAPHIE}

AGLIETTA, M., BRENDER, A. et COUDERT, V., Globalisation financière :l'Aventure obligée, Economica, Paris, 1990.

ATTALI, J., Lignes d'horizon, Fayard, Paris, 1990.

BRADFORD JR, C., (dir.), Options stratégiques pour l'Amérique latine dans les années 90, OCDE, Paris, 1992.

CEPAL, Transformacion productiva con equidad, Nations Unies, Santiago, 1990.

CEPAL, Balance preliminar de la economia de America Latina y del Caribe, Santiago, décembre 1991.

CEPAL, Equidad y transforcion productiva : un enfoque integrado, Nations Unies, Santiago, 1992.

CEPII, Économie mondiale 1990-2000 : l'impératif de croissance, Economica, Paris, 1992.

COMMISSION DES COMMUNAUTES EUROPEENNES, « Les relations de la Communauté européenne avec l'Amérique latine », Europe Information, Bruxelles, décembre 1989.

COMMISSION DES COMMUNAUTES EUROPEENNES, « Orientations pour la coopération avec les pays en développement d'Amérique latine et d'Asie », Bruxelles, juin 1990.

ECK, J.F., Le commerce mondial de produits agricoles au XX ${ }^{\text {ème }}$ siècle, Ed. Eyrolles, Paris, 1992. 
EURAL, Lavulnerabilidad exterior de America Latina y Europa, Grupo Editor Latinoamericano, BuenosAires, 1985.

FUENTES, J.A., « Compatibilidad entre la integracion subregional y la hemisferica », Revista de la Cepal, $\mathrm{n}^{\circ}$ 45, Santiago, décembre 1991.

GRANDA, G., " Amérique latine-Communauté européenne : une coopération en marche », Cahiers CERCAL, $\mathrm{n}^{\circ}$ 4, Bruxelles, juillet 1991.

GRANDI, J., « L'impact du Marché unique européen sur l'Amérique latine : obstacles et opportunités », Cahiers CERCAL, Bruxelles, janvier 1992.

HEINE, J., « Cooperacion o divergencia ? Hacia una nueva agenda en las relaciones europeoIatinoamericanas », CEPAL, LC/R. 929, Santiago, octobre 1990.

HENRY, G.M., Dynamique du commerce international : nouveau protectionnisme et libreéchange, Ed. Eyrolles, Paris 1992.

IRELA, « La Iniciativa para las Americas y las relaciones europeolatinoamericanas », Dossier n³1, Madrid, mai 1991.

IRELA, « El Mercado Unico Europeo y America Latina », Dossier n³4, Madrid, septembre 1991.

IZAM, M., « Europa 92 y la economia latinoamericana », Revista de la Cepal, n 43, Santiago, avril 1991.

LOWENTHAL, P., « Ouverture économique et intégration régionale stratégies complémentaires pour l'Amérique latine », Cahiers CERCAL, n 11, Bruxelles, septembre 1992.

OMINAMI, C. (dir.). La tercera revolucion indus trial, Grupo Editor Latinoamericano, Buenos-Aires, 1986.

SELA, Relaciones economicas internacionales de America Latina, Ed. Nueva Sociedad, Caracas, 1987.

VAN KLAVEREN, A., « Les relations Europe-Amérique latine : entre l'illusion et le réalisme », Cahiers CERCAL, $\mathrm{n}^{\circ}$ 7, Bruxelles, janvier 1992.

\section{NOTES}

1. CEPII, Économie mondiale 1990-2000 : l'impératif de croissance, Economica, Paris, 1992, p. 381.

2. Cfr. Attali, J., Lignes d'horizon, Ed. Fayard, Paris, 1990, ch. 2.

3. CEPII. op.cit., p. 14.

4. Aglietta, M., Brender, A. et Coudert, V., Globalisation financière : l'aventure obligée, Economica, Paris, 1990.

5. Chiffres cités par la presse économique internationale en septembre 1992.

6. Galbraith. J.K., Brève histoire de l'euphorie financière, Seuil, Paris, 1992.

7. Cfr. Henry, G., Dynamique du commerce international : nouveau protectionnisme et libre échange,Ed. Eyrolles, Paris, 1992, pp. 82-93.

8. Selon les chiffres de la Banque Mondiale, Rapport sur le développement dans le monde 1991, Washington D.C., 1991 (indicateurs du développement, tableaux 1 et 3).

9. Sur ce concept, cfr. M. Benedetti, «Los dos capitalismos », El Pais, Madrid, 30/12/1991.

10. CEPAL, Transjormacion productiva con equidad, Santiago, 1990, ch. 1.

11. IRELA, «El Mercado Unico Europeo y America Latina », Dossier $n^{\circ} 34$, Madrid, septembre 1991, (tableau 3). 
12. IRELA, «La Iniciativa para las Americas y las relaciones europeo-latinoamericanas », Dossier $\mathrm{n}^{\circ}$ 31, Madrid, mai 1991.

13. Cfr. l'excellente analyse de J.F. Eck, Le commerce mondial des produits agricoles auXX ${ }^{\text {ème }}$ siècle, Ed. Eyrolles, Paris, 1992, pp. 134-139.

14. Relevant de six pays : Allemagne, France, Italie, Pays-Bas, Royaume-Uni et Suisse.

15. IRELA, « La Iniciativa ... » , op.cit., p. 48.

16. Cfr. A. Suarez, «La concurrence interbancaire et la gestion de la crise de l'endettement latino-américain », Cahiers CERCAL, n6, Bruxelles, novembre 1991.

17. Ibid.

18. IRELA, « La Iniciativa ... «, op.cit., p. 55.

19. Ibid., p. 54.

20. El Financiero, Mexico, 30/06/1992.

21. Sur ce thème, voir G. Ruiz-Gimenez, "Nouvelles politiques européennes de coopération au développement de l'Amérique latine ", Cahiers CERCAL, n³, Bruxelles, mai 1991 et G. Granda, "Amérique latine-Communauté européenne : une coopération en marche », Cahiers CERCAL, n4, Bruxelles, juillet 1991.

22. Cfr. P. Lowenthal, "Ouverture économique et intégration régionale: stratégies complémentaires pour l'Amérique latine ", Cahiers CERCAL, n¹1, Bruxelles, septembre 1992.

23. IRELA, «El Mercado Unico Europeo y America Latina », op.cit.

24. Cfr. CEPAL, Transjormacion productiva con equidad, op.cit.

25. CEPAL, Balance preliminar de la economia de America Latina y del Caribe, Santiago, décembre 1991, p. 19. Cette tendance s'est confirmée et accentuée en 1992.

26. Ibid., p. 21.

27. Ce problème est analysé dans l'article de M. Ossandon, dans cette même revue.

\section{RÉSUMÉS}

The author presents an analysis of the main tendencies that characterise the recent development of the world economy : productive internationalisation, market globalisation, regionalisation, in order to situate in this context the evolution of economic relations between Latin America and the European Community for the past ten years. On the basis of this interpretation, the author analyses questions that, according to him, should compose the economic agenda of relations between the two regions for the coming years: the conditions of access of Latino-American exported goods to the Community market, the foreign debt, the flow of investments, the impact of privileged relations with the countries of Eastem Europe and environmental matters. For all these questions, there appears to be convergence of interests that can be materialized as groups of cooperation, but also differences liable of declining into more or less declared conflicts. Finally, an essential question refers to the role that Europe will or will not want to play in the process of Latin America's reinsertion in the world economy, either by accepting the consequences of the struggle for power expressed through the "market rules", or by recognizing Latin America as having the status of a responsible and active partner that it claims on the international scene. 


\section{AUTEUR}

\section{MARC RIMEZ}

Institut d'études du développement, Université catholique de Louvain 